\title{
Investigation of quantum roulette
}

\author{
S. Salimi *, M.M. Soltanzadeh ${ }^{\dagger}$ \\ Department of Physics, University of Kurdistan, Sanandaj 51664, Iran.
}

December 1, 2018

\begin{abstract}
In this paper, by using permutation matrices as a representation of symmetric group $S_{N}$ and Fourier matrix, we investigate quantum roulette with an arbitrary $N$-state. This strategy, which we introduce, is general method that allows us to solve quantum game for an arbitrary $N$-state. We consider the interaction between the system and its environment and study the effect of the depolarizing channel on this strategy. Finally, as an example we employ this strategy for quantum roulette with $N=3$.
\end{abstract}

Keywords: Quantum roulette, Quantum game, Quantum strategy and coin tossing.

PACs Index: 03.65.Ud

${ }^{*}$ Corresponding author: E-mail:shsalimi@uok.ac.ir

†E-mail:msoltanzadeh@uok.ac.ir 


\section{Introduction}

Recent research in quantum computation, communication and cryptography has focussed on the physical aspect of information. In the most of the cases quantum description of the system provides advantages over the classical situation. For example, Simon's quantum algorithm [1] to identify the period of a function chosen by a oracle is more efficient than any deterministic or probabilistic algorithm, Shor's polynomial time quantum algorithm [2] for factoring and the quantum protocols for key distribution devised by Bennet and Brassard [3] and Ekert [4] are qualitatively more secure against eavesdropping than any classical cryptographic system.

Game theory is the study of decision making in conflict situation. Recently, quantum game theory $[5,6,7,8,9,10,11,12,13,14,15,16]$ has been investigated, which discusses versions of some classical game [17] where new rules that make explicit use of quantum mechanics lead to new solutions. D.A. Meyer [5] demonstrated that in a classical two-person zero-sum strategic game, if one person adopts a quantum strategy, then he has a better chance of winning the game. And based on these work, Xiang-Bin Wang, L.C. Kwek et al. [18] extended this case by replacing the coin which has only two possible states (namely head and tail) with a roulette with $N=2^{m}(m=1,2, \ldots)$ states, and concluded that quantum strategies can also be more successful than classical ones; Jing-Ling Chen, L.C. Kwek and C.H. Oh [19] studied noisy quantum game. In this paper we investigate quantum roulette with arbitrary $N$ states. To solve this problem we use permutation matrices as a representation of symmetric group $S_{N}$ and Fourier matrix. This strategy is general and one can generalize easily to any integer $N$ which for example we employ it for quantum roulette with $N=3$. Also, in the end we consider the interaction between the system and its environment and investigate depolarizing channel on this strategy.

The organization of the paper is as follows. In Section 2, we give a brief outline of a quantum coin-tossing game. In Section 3, we generalize a quantum coin-tossing by replacing the coin, 
which has only two possible state, with a roulette with arbitrary $N$ state and we employ this strategy for a quantum game with $N=3$, also in the end of this section we investigate the effect of decoherence channel, depolarizing channel, on this strategy. The paper is ended with a brief conclusion.

\section{A quantum coin-flipping game}

In this section we review Meyer's strategies to play with a single coin. The classical coin has only two possible states, head and tail. It is natural to define a two-dimensional Hilbert space $H_{2}$ with basis $|H\rangle$ and $|T\rangle$ (the symbols $H$ and $T$ denote head and tail, respectively) which could be represented by:

$$
|H\rangle=\left(\begin{array}{l}
1 \\
0
\end{array}\right), \quad|T\rangle=\left(\begin{array}{l}
0 \\
1
\end{array}\right) .
$$

The player strategies represent by matrices of $2 \times 2$

$$
N=\left(\begin{array}{ll}
1 & 0 \\
0 & 1
\end{array}\right), \quad F=\left(\begin{array}{ll}
0 & 1 \\
1 & 0
\end{array}\right)
$$

correspond to not flipping and flipping the coin, respectively. Owing to $N$ and $F$, we can construct a density matrix $D$ as follows:

$$
D=\frac{1}{2}(N+F)=\frac{1}{2}\left(\begin{array}{cc}
1 & 1 \\
1 & 1
\end{array}\right), \quad \operatorname{Trace}(D)=1 .
$$

It is easy to verify that $D$ commutes with $N$ and $F$, i.e.,

$$
[N, D]=[N, F]=0
$$

With due attention to the unitary of $N$ and $F$, from above equation we would have an identity

$$
D=(1-p) N D N^{\dagger}+p F D F^{\dagger}
$$


which is independent upon the parameter $p \in[0,1]$ which is probability that the player flips the coin.

The general pure state if a quantum coin is

$$
|\psi\rangle=\cos \left(\frac{\theta}{2}\right)|H\rangle+e^{i \phi} \sin \left(\frac{\theta}{2}\right)|T\rangle
$$

where corresponding density matrix is given by

$$
\rho=|\psi\rangle\langle\psi|=\frac{1}{2}\left(\begin{array}{cc}
1+\cos (\theta) & e^{-i \phi} \sin (\theta) \\
e^{i \phi} \sin (\theta) & 1-\cos (\theta)
\end{array}\right) .
$$

Now Alice and Bob come to play a coin-tossing game, such that Alice utilize a classical probabilistic strategy in which she flips the coin with probability $p$, but Bob could control this game by quantum strategies. The game accomplish in four step as follows:

Step1: Let us the initial state of the coin which is placed by Alice be $\left|\psi_{0}\right\rangle$, thus its density matrix is given by

$$
\rho_{0}=\left|\psi_{0}\right\rangle\left\langle\psi_{0}\right|
$$

Step2: Bob acts on coin by a quantum strategy (unitary transformation $U_{1}$ ), then the state of the coin becomes

$$
\rho_{1}=U_{1} \rho_{0} U_{1}^{\dagger}=D
$$

where

$$
\begin{aligned}
& U_{1}=\left(\left|\lambda_{0}\right\rangle,\left|\lambda_{1}\right\rangle\right)=\frac{1}{\sqrt{2}}\left(\begin{array}{cc}
1 & 1 \\
1 & -1
\end{array}\right), \text { if } \rho_{0}=|H\rangle\langle H| \\
& U_{1}=\left(\left|\lambda_{1}\right\rangle,\left|\lambda_{0}\right\rangle\right)=\frac{1}{\sqrt{2}}\left(\begin{array}{cc}
1 & 1 \\
-1 & 1
\end{array}\right), \text { if } \rho_{0}=|T\rangle\langle T| .
\end{aligned}
$$

In the above equation the $\left|\lambda_{0}\right\rangle$ and $\left|\lambda_{1}\right\rangle$ are eigenvectors of density matrix $D$ with eigenvalues $\lambda_{0}=1$ and $\lambda_{1}=0$, respectively. 
Step3: Alice continues to play with a classical strategy, namely, Alice employs a convex sum of unitary (deterministic) transformation, i.e., he either flips the coin using the transformation $F$ with probability $p$ or lets the coin rest in its original state (using the identity transformation $N)$ with probability $(1-p)$. Thus by using (2-5), one can know that Alice's classical strategy does not change the density matrix of the coin, i.e.,

$$
\rho_{2}=(1-p) N \rho_{1} N^{\dagger}+p F \rho_{1} F^{\dagger}=\rho_{1}=D
$$

Step4: Finally, Bob could control the game by an appropriate unitary transformation, $U_{2}=$ $U_{1}^{\dagger}$, i.e., because the density matrix is still $D$ he can adopt an unitary matrix $U_{2}$ to transform it into the state which he wants to.

\section{Investigation of quantum roulette with arbitrary $N$ state}

In this section we generalize a quantum coin-tossing by replacing the coin, which has only two possible state (namely head and tail), with a roulette with $N$ state.

Here we consider Hilbert space $H$ as a quantum roulette with $N$-dimensional and we indicate $N$ basis of quantum roulette with $|k\rangle$ for $k=1,2, \ldots, N$, where could be represented by the following matrices:

$$
|1\rangle=\left(\begin{array}{c}
1 \\
0 \\
\vdots \\
0 \\
0
\end{array}\right), \quad|2\rangle=\left(\begin{array}{c}
0 \\
1 \\
\vdots \\
0 \\
0
\end{array}\right), \quad \cdots \quad|N\rangle=\left(\begin{array}{c}
0 \\
0 \\
\vdots \\
0 \\
1
\end{array}\right) .
$$

Thus, the roulette has $N$ state which Alice has a choice $N$ ! possible flips corresponding to all the possible permutation of the state $\{1,2,3, \ldots, N\}$ to itself, where is called symmetric 
group $S_{N}$. Therefore the explicit matrix form of operators are permutation matrices as a representation of symmetric group $S_{N}$. By using Ref.[20], if $\pi \in S_{N}$ then the permutation matrix $X(\pi)=\left(x_{i, j}\right)_{N \times N}$ is defined as follows:

$$
x_{i, j}=\left\{\begin{array}{cc}
1 & \text { if } \pi(j)=i \\
0 & \text { otherwise }
\end{array}\right.
$$

that it contains only zeros and ones, with unique one in every row and column. Now we let $X^{i}$, for $i=0,1,2, \ldots N !-1$, as permutation operators, then we can construct the density matrix $D$

$$
D=\frac{1}{N !} \sum_{i=0}^{N !-1} X^{i}=\frac{1}{N} J_{N}
$$

where $J_{N}$ is $N \times N$ matrix with all matrix elements equal to 1 .

Due to the matrices $X^{i},(i=0,1,2,3, \ldots, N !-1)$ have unique one in every row and column, one can prove that $D$ commutes with $X^{i},(i=0,1,2, \ldots, N !-1)$, i.e.,

$$
\left[D, X^{i}\right]=0 \quad \text { for }(i=0,1,2, \ldots, N !-1)
$$

Therefore we have

$$
D=\left(1-\sum_{i=1}^{N !-1} p_{i}\right) X^{0} D X^{0 \dagger}+\sum_{i=1}^{N !-1} p_{i} X^{i} D X^{i \dagger},
$$

which is independent on parameter $p_{i}$ and $X^{0}$ is identity matrix or not flipping operator.

Now to obtain the unitary transformation of Bob strategy we need eigenvector of density matrix $D$. The density matrix $D$ is circulant matrix [21]. An important property of circulant matrices is that they (unitarily) diagonalizable by the Fourier matrix

$$
F=\frac{1}{\sqrt{n}} V(\omega)
$$


where $\omega=e^{2 \pi i / n}$ and $V(\omega)$ is the Vandermonde matrix defined as

$$
V(\omega)=\left(\begin{array}{ccccc}
1 & 1 & 1 & \cdots & 1 \\
1 & \omega & \omega^{2} & \cdots & \omega^{n-1} \\
1 & \omega^{2} & \omega^{4} & \cdots & \omega^{2(n-1)} \\
\vdots & \vdots & \vdots & \vdots & \vdots \\
1 & \omega^{n-1} & \omega^{2(n-1)} & \cdots & \omega^{(n-1)^{2}}
\end{array}\right) .
$$

Let the $k$-th column vector of $V(\omega)$ be denoted by $\left|\omega_{k}\right\rangle, k=0,1,2, \ldots, n-1$. It is easy to verify that $F$ is unitary (i.e., $F^{-1}=F^{\dagger}$ ), since the Vandermonde matrix obeys $V(\omega)^{-1}=V\left(\omega^{-1}\right)$. Thus, for a $n$-square circulant matrix $C$ we have

$$
F^{\dagger} C F=\operatorname{diag}\left(f\left(\omega^{0}\right), f\left(\omega^{1}\right), \ldots, f\left(\omega^{(n-1)}\right)\right)
$$

where $f(\mu)=c_{0}+c_{1} \mu^{1}+c_{1} \mu^{2}+\ldots+c_{1} \mu^{(n-1)}$ and $\left(c_{0}, c_{1}, \ldots, c_{n-1}\right)$ are matrix elements of $C$.

In this case, the matrix elements are fixed and equal to $\frac{1}{N}$, i.e., $c_{0}=c_{1}=\ldots=c_{N-1}=\frac{1}{N}$, where the quantity of $n$ is $N$. Therefore, by using Eq.(3-18) and above definition for $f$, one can obtain eigenvalues of density matrix of $D$

$$
\lambda_{0}=f\left(\omega^{0}\right)=1, \quad \lambda_{1}=f\left(\omega^{1}\right)=\lambda_{2}=f\left(\omega^{2}\right)=\cdots=\lambda_{N-1}=f\left(\omega^{N-1}\right)=0,
$$

with the eigenvectors as

$$
\left|\lambda_{k}\right\rangle=\frac{1}{\sqrt{N}}\left|\omega_{k}\right\rangle, \quad \text { for } k=0,1,2, \ldots, N-1
$$

where $\left|\omega_{k}\right\rangle$ denote the $k$-th column vector of $V(\omega)$.

In the above calculation we used formula:

$$
\sum_{l=0}^{N-1} \omega^{l k}=0, \quad \text { for } \omega=e^{\frac{2 \pi i}{N-1}} .
$$

Now we are in the position to derive the unitary transformation of Bob strategy as

$$
T_{1}=\left(\left|\lambda_{0}\right\rangle,\left|\lambda_{1}\right\rangle, \ldots,\left|\lambda_{N-1}\right\rangle\right)=\frac{1}{\sqrt{N}} V(\omega)
$$




$$
\begin{aligned}
& T_{2}=\left(\left|\lambda_{N-1}\right\rangle,\left|\lambda_{0}\right\rangle, \ldots,\left|\lambda_{N-2}\right\rangle\right)=\frac{1}{\sqrt{N}}\left(\begin{array}{ccccc}
1 & 1 & 1 & \cdots & 1 \\
\omega^{N-1} & 1 & \omega & \cdots & \omega^{N-2} \\
\omega^{2(N-1)} & 1 & \omega^{2} & \cdots & \omega^{2(N-2)} \\
\vdots & \vdots & \vdots & \vdots & \vdots \\
\omega^{(N-1)^{2}} & 1 & \omega^{(N-1)} & \cdots & \omega^{(N-1)(N-2)}
\end{array}\right) \\
& T_{N}=\left(\left|\lambda_{1}\right\rangle,\left|\lambda_{2}\right\rangle, \ldots,\left|\lambda_{N-1}\right\rangle,\left|\lambda_{0}\right\rangle\right)=\frac{1}{\sqrt{N}}\left(\begin{array}{ccccc}
1 & 1 & \cdots & 1 & 1 \\
\omega & \omega^{2} & \cdots & \omega^{N-1} & 1 \\
\omega^{2} & \omega^{4} & \cdots & \omega^{2(N-1)} & 1 \\
\vdots & \vdots & \vdots & \vdots & \vdots \\
\omega^{(N-1)} & \omega^{2(N-1)} & \cdots & \omega^{(N-1)^{2}} & 1
\end{array}\right) .
\end{aligned}
$$

Now we consider quantum roulette game with arbitrary $N$ states and we discuss Bob and Alice how to control the game by quantum and classical strategies, respectively. We can consider quantum roulette as Hilbert space with $N$-dimension. The initial states which can adopt are

$$
|1\rangle\left\langle 1\left|=\left(\begin{array}{ccccc}
1 & 0 & \cdots & 0 & 0 \\
0 & 0 & \cdots & 0 & 0 \\
0 & 0 & \cdots & 0 & 0 \\
\vdots & \vdots & \vdots & \vdots & \vdots \\
0 & 0 & \cdots & 0 & 0
\end{array}\right), \quad\right| 2\right\rangle\langle 2|=\left(\begin{array}{ccccc}
0 & 0 & \cdots & 0 & 0 \\
0 & 1 & \cdots & 0 & 0 \\
0 & 0 & \cdots & 0 & 0 \\
\vdots & \vdots & \vdots & \vdots & \vdots \\
0 & 0 & \cdots & 0 & 0
\end{array}\right),
$$




$$
|N\rangle\langle N|=\left(\begin{array}{ccccc}
0 & 0 & \cdots & 0 & 0 \\
0 & 0 & \cdots & 0 & 0 \\
0 & 0 & \cdots & 0 & 0 \\
\vdots & \vdots & \vdots & \vdots & \vdots \\
0 & 0 & \cdots & 0 & 1
\end{array}\right),
$$

where one can obtain above density matrices form similarity transformation i.e., $|k\rangle\langle k|=$ $T_{k}^{\dagger} D T_{k}$ for $k=1,2, \ldots, N$.

We suppose that the initial state of the roulette is $\left|\psi_{0}\right\rangle$ and its density matrix is $\rho_{0}=$ $\left|\psi_{0}\right\rangle\left\langle\psi_{0}\right|$. Then Alice and Bob will play a roulette game. During the game, Bob adopts a quantum strategy by using a unitary matrix to act on the coins while Alice adopts the usual classical probabilistic strategy. Now we have:

Step1: Alice places the roulette on one box such that the state of the coins is known by both Alice and Bob.

Step2: Bob uses a unitary transformation $U_{1}$ to act on the roulette, such that, if the initial state is $|1\rangle,|2\rangle, \ldots$, or $|N\rangle$ the unitary transformation $U_{1}$ is corresponding $T_{1}, T_{2}, \ldots$, or $T_{N}$, then the state of the coins become

$$
\rho_{1}=U_{1} \rho_{0} U_{1}^{\dagger}=D
$$

Step3: Alice continues to play by employing classical strategy, namely she perhaps changes the state of the roulette using the permutation matrices $X^{i}(i=0,1,2, \ldots, N !-1)$ with the probability $p_{i}$. Thus, at the end of Alice's play, the state of the roulette is described by the density matrix

$$
\rho_{2}=\left(1-\sum_{i=1}^{N !-1} p_{i}\right) X^{0} \rho_{1} X^{0 \dagger}+\sum_{i=1}^{N !-1} p_{i} X^{i} \rho_{1} X^{i \dagger}=\left(1-\sum_{i=1}^{N !-1} p_{i}\right) X^{0} D X^{0 \dagger}+\sum_{i=1}^{N !-1} p_{i} X^{i} D X^{i \dagger}=D .
$$

Step4: Bob plays with the roulette by using the unitary transformation $U_{2}$ so that the density 
matrix of final state of the coins is given by

$$
\rho_{3}=U_{2} \rho_{2} U_{2}^{\dagger}
$$

Here Bob can get every arbitrary state with choice $U_{2}=U_{1}^{\dagger}$, for example if he wants to get the state $|1\rangle$ then $U_{2}=T_{1}^{\dagger}$; and $|1\rangle, U_{2}=T_{2}^{\dagger}$ and so forth. Therefore, Bob can control the game by the above quantum strategies.

This strategy is general method and one can employ easily to any integer $N$. For example we employ it for quantum roulette with $N=3$. In this case, there is a choice of 3 ! possible flips corresponding to all group symmetric $S_{3}$. Thus the basis and permutation matrices $[20]$ are

$$
\begin{gathered}
|1\rangle=\left(\begin{array}{l}
1 \\
0 \\
0
\end{array}\right), \quad|2\rangle=\left(\begin{array}{l}
0 \\
1 \\
0
\end{array}\right), \quad|3\rangle=\left(\begin{array}{l}
0 \\
0 \\
1
\end{array}\right), \\
X^{0}=\left(\begin{array}{lll}
1 & 0 & 0 \\
0 & 1 & 0 \\
0 & 0 & 1
\end{array}\right), \quad X^{1}=\left(\begin{array}{lll}
0 & 1 & 0 \\
1 & 0 & 0 \\
0 & 0 & 1
\end{array}\right), \quad X^{2}=\left(\begin{array}{lll}
0 & 0 & 1 \\
0 & 1 & 0 \\
1 & 0 & 0 \\
1 & 0 & 0
\end{array}\right), \quad X^{4}=\left(\begin{array}{lll}
0 & 0 & 1 \\
1 & 0 & 0 \\
0 & 0 & 1 \\
0 & 1 & 0
\end{array}\right), \quad X^{5}=\left(\begin{array}{lll}
0 & 1 & 0 \\
0 & 0 & 1 \\
1 & 0 & 0
\end{array}\right),
\end{gathered}
$$

respectively. Then by using the Eq. (3-13), we can construct the density matrix $D$ as

$$
D=\frac{1}{3 !} \sum_{i=0}^{5} X^{i}=\frac{1}{3} J_{3}=\frac{1}{3}\left(\begin{array}{ccc}
1 & 1 & 1 \\
1 & 1 & 1 \\
1 & 1 & 1
\end{array}\right)
$$


The Fourier matrix (3-16) and unitary transformation $T_{i}(i=1,2,3)$ Eq. (3-22) are given by

$$
\begin{aligned}
& F=\frac{1}{\sqrt{3}}\left(\begin{array}{ccc}
1 & 1 & 1 \\
1 & \omega & \omega^{2} \\
1 & \omega^{2} & \omega^{4}
\end{array}\right) \\
& T_{1}=F, \quad T_{2}=\frac{1}{\sqrt{3}}\left(\begin{array}{ccc}
1 & 1 & 1 \\
\omega^{2} & 1 & \omega \\
\omega^{4} & 1 & \omega^{2}
\end{array}\right), \quad T_{3}=\frac{1}{\sqrt{3}}\left(\begin{array}{ccc}
1 & 1 & 1 \\
\omega & \omega^{2} & 1 \\
\omega^{2} & \omega^{4} & 1
\end{array}\right) \text {, }
\end{aligned}
$$

for $\omega=e^{\frac{2 \pi i}{3}}$.

For initial state $\left|\psi_{0}\right\rangle$, i.e., $|1\rangle,|2\rangle$ or $|3\rangle$, the unitary transformation $U_{1}$ is corresponding $T_{1}, T_{2}$, or $T_{3}$. For example we consider initial state $\left|\psi_{0}\right\rangle=|2\rangle$ then the density matrix $\rho_{0}$ is

$$
\rho_{0}=|2\rangle\langle 2|=\left(\begin{array}{ccc}
0 & 0 & 0 \\
0 & 1 & 0 \\
0 & 0 & 0
\end{array}\right) \text {. }
$$

Thus Bob rotates the initial density matrix as

$$
\rho_{1}=U_{1} \rho_{0} U_{1}^{\dagger}=T_{2} \rho_{0} T_{2}^{\dagger}=D
$$

Alice continues to play by employing classical strategy, then by using Eq. (3-25) the density matrix takes the form

$$
\begin{gathered}
\rho_{2}=\left(1-\left(p_{1}+p_{2}+p_{3}+p_{4}+p_{5}\right)\right) X^{0} \rho_{1} X^{0 \dagger}+p_{1} X^{1} \rho_{1} X^{1 \dagger}+p_{2} X^{2} \rho_{1} X^{2 \dagger} \\
+p_{3} X^{3} \rho_{1} X^{3 \dagger}+p_{4} X^{4} \rho_{1} X^{4 \dagger}+p_{5} X^{5} \rho_{1} X^{5 \dagger}=D .
\end{gathered}
$$

Bob can always control the final state by using the unitary matrix $U_{2}=T_{1}^{\dagger}, T_{2}^{\dagger}, T_{3}^{\dagger}$, i.e., if he want state $|1\rangle$

$$
\rho_{3}=T_{1}^{\dagger} \rho_{2} T_{1}=\left(\begin{array}{ccc}
1 & 0 & 0 \\
0 & 0 & 0 \\
0 & 0 & 0
\end{array}\right)=|1\rangle\langle 1|,
$$


if he want state $|2\rangle$

$$
\rho_{3}=T_{2}^{\dagger} \rho_{2} T_{2}=\left(\begin{array}{ccc}
0 & 0 & 0 \\
0 & 1 & 0 \\
0 & 0 & 0
\end{array}\right)=|2\rangle\langle 2|,
$$

if he want state $|3\rangle$

$$
\rho_{3}=T_{3}^{\dagger} \rho_{2} T_{3}=\left(\begin{array}{ccc}
0 & 0 & 0 \\
0 & 0 & 0 \\
0 & 0 & 1
\end{array}\right)=|3\rangle\langle 3| .
$$

\subsection{Noisy quantum game}

In this subsection we investigate the interaction between the quantum game and its environment. The interaction between the system and its environment introduce decoherence to the system, which is a process of the undesired correlation between the system and the environment when the system evolves. Therefore, the communication accomplished under noisy channels [22] may not be faithful because the receiver may obtain partial or corrupted information different from sender's information. The quantum noise process is represented by mapping $\rho \Longrightarrow \mathcal{S}(\rho)$, where $\mathcal{S}$ is a super-operator [23] that makes the initial state $\rho$ evolve to the final state $\mathcal{S}(\rho)$. In general, the communication process of an open system can be represented by the operator-sum representation

$$
\mathcal{S}(\rho)=\sum_{k} E_{k} \rho E_{k}^{\dagger}
$$

where $E_{k}$ are kraus operator elements for the super operation $\mathcal{S}$, and are trace-preserving, $\sum_{k} E_{k} E_{k}^{\dagger}=I$. There are several decoherence channels which for example we consider the depolarizing channel. For a qubit system the kraus operators of depolarizing channel are

$$
E_{0}=\sqrt{1-r} I, \quad E_{1}=\sqrt{\frac{r}{3}} \sigma_{x}, \quad E_{2}=\sqrt{\frac{r}{3}} \sigma_{y}, \quad E_{3}=\sqrt{\frac{r}{3}} \sigma_{z}
$$

where $\left(\sigma_{x}, \sigma_{y}, \sigma_{z}\right)$ is the set of Pauli matrices. This channel acts on qubits by phase flips, amplitude flips or combinations of both applied with probability $r / 3$ each. More generally, for 
a quantum $d$-ary digit (a qudit), is a $d$-dimensional Hilbert space $H$ with orthonormal basis as $(|0\rangle,|1\rangle, \ldots,|d-1\rangle)$, we can define a depolarizing channel [24] as follows:

$$
E_{0}=\sqrt{1-r} I, \quad E_{k}=\sqrt{\frac{r}{d^{2}-1}} M_{i, j}, \quad i, j \in \mathcal{F}_{d}
$$

where $\mathcal{F}_{d}$ is a finite field and $M_{i, j}$ is defined as $\left\{M_{i, j}=Y^{i} Z^{j}, i, j \in \mathcal{F}_{d}\right\}$ such that

$$
Y|l\rangle=|(l-1) \bmod \mathrm{d}\rangle, \quad Z|l\rangle=\omega^{l}|l\rangle
$$

and $\omega=e^{\frac{2 \pi i}{d}}$ is a primitive $d$ th root of unity.

Now we consider the previous example (i.e., $N=3$ ) with decoherence model. In this case the depolarizing channel has $E_{k}$ (Eq. 3-38) represented by

$$
\begin{gathered}
E_{0}=\sqrt{1-r} I, \quad E_{1}=\sqrt{\frac{r}{8}} Y, \quad E_{2}=\sqrt{\frac{r}{8}} Z, \quad E_{3}=\sqrt{\frac{r}{8}} Y^{2}, \quad E_{4}=\sqrt{\frac{r}{8}} Y Z, \\
E_{5}=\sqrt{\frac{r}{8}} Y^{2} Z, \quad E_{6}=\sqrt{\frac{r}{8}} Y Z^{2}, \quad E_{7}=\sqrt{\frac{r}{8}} Y^{2} Z^{2}, \quad E_{8}=\sqrt{\frac{r}{8}} Z^{2},
\end{gathered}
$$

with

$$
Y=\left(\begin{array}{ccc}
0 & 1 & 0 \\
0 & 0 & 1 \\
1 & 0 & 0
\end{array}\right), \quad Z=\left(\begin{array}{ccc}
1 & 0 & 0 \\
0 & \omega & 0 \\
0 & 0 & \omega^{2}
\end{array}\right), \quad \omega=e^{\frac{2 \pi i}{3}} .
$$

For simplicity, we consider only the interaction between the system with its environment in the first step. Therefore, we have

Step1. The initial state $\rho_{0}=|2\rangle\langle 2|$ is decohered by depolarizing channel where become

$$
\rho_{0}^{\prime}=\sum_{k=0}^{8} E_{k} \rho_{0} E_{k}^{\dagger}=\frac{r}{8}\left(\begin{array}{ccc}
1 & \alpha+e^{\frac{-2 \pi i}{3}} & 1 \\
\alpha+e^{\frac{2 \pi i}{3}} & \frac{8}{r}-7-\alpha & \alpha+e^{\frac{2 \pi i}{3}} \\
1 & \alpha+e^{\frac{-2 \pi i}{3}} & 1
\end{array}\right)
$$

where $\alpha=2 \sqrt{2 r(1-r)}$.

Step2. Bob the applies the unitary transformation $U_{1}=T_{2}$ as

$$
\rho_{1}=T_{2} \rho_{0} T_{2}^{\dagger}=\left(\begin{array}{ccc}
\beta & \xi & \xi \\
\xi^{\star} & \eta & \eta \\
\xi^{\star} & \eta & \eta
\end{array}\right)
$$


with

$$
\beta=1 / 24(8+(-5+3 \alpha) r), \quad \xi=1 / 48(16+(-19-3 i \sqrt{3}) r), \quad \eta=1 / 24(8-(5+3 \alpha) r) .
$$

In this case we see that the $\rho_{1}$ is not equal with $D$.

Step3. Now the Alice does not delay (i.e., the system is not decohered) and continues to play by employing classically strategy, then we have

$$
\rho_{2}=\left(1-\sum_{i=1}^{5} p_{i}\right) X^{0} \rho_{1} X^{0 \dagger}+\sum_{i=1}^{5} p_{i} X^{i} \rho_{1} X^{i \dagger} \neq D
$$

Step4. Then Bob implements the unitary transformation $U_{2}=T_{2}^{\dagger}$. In this case the density matrix of final state is

$$
\rho_{3}=T_{2}^{\dagger} \rho_{2} T_{2}=\left(\begin{array}{ccc}
\rho_{11} & \rho_{12} & \rho_{13} \\
\rho_{21} & \rho_{22} & \rho_{23} \\
\rho_{31} & \rho_{32} & \rho_{33}
\end{array}\right)
$$

where

$$
\begin{gathered}
\rho_{11}=-\frac{r}{16}\left(-2+2 p_{1}+(3+i \sqrt{3}) p_{4}+3 p_{5}-i \sqrt{3} p_{5}\right) \\
\rho_{12}=\frac{r e^{2 i \pi / 3}}{16}\left(-2+\alpha-i \sqrt{3} \alpha+(5-i \sqrt{3}+(-1+3 i \sqrt{3}) \alpha) p_{1}+(3+i \sqrt{3}-3 \alpha+i \sqrt{3} \alpha) p_{2}+\right. \\
\left.(3-i \sqrt{3}+2 i \sqrt{3} \alpha) p_{4}+(3+i \sqrt{3}-3 \alpha+i \sqrt{3} \alpha) p_{5}\right) \\
\left.\rho_{13}=\frac{r e^{2 i \pi / 3}}{16 \sqrt{3}}\left(-3 i-\sqrt{3}+(9 i+\sqrt{3}) p_{1}+3(i+\sqrt{3}) p_{2}\right)\right) \\
\rho_{21}=-\frac{r}{16 \sqrt{3}}\left(-3 i+\sqrt{3}-2 \sqrt{3} \alpha+(6 i-4 \sqrt{3}+(3 i+5 \sqrt{3}) \alpha) p_{1}+3(2 i+(-i+\sqrt{3}) \alpha) p_{2}+\right. \\
\left.((6-3 \alpha) i+3 \sqrt{3} \alpha) p_{4}+(3(1+\alpha) i+3 \sqrt{3}(-1+\alpha)) p_{5}\right) \\
\rho_{22}=\frac{1}{8}(-8+(7+\alpha) r)\left(-1+p_{1}\right) \\
\frac{r}{16 \sqrt{3}}\left(-3 i+\sqrt{3}-2 \sqrt{3} \alpha+(9 i-\sqrt{3}+(-3 i+5 \sqrt{3}) \alpha) p_{1}+3(i-\sqrt{3}+(i+\sqrt{3}) \alpha) p_{2}+\right. \\
\left.\left.(3(1+\alpha) i+3 \sqrt{3}(-1+\alpha)) p_{4}+(6(3-\alpha) i+3 \sqrt{3} \alpha) p_{5}\right)\right) \\
\rho_{31}=\frac{r}{16}\left(2+(-5+\sqrt{3} i) p_{1}+(-3-i \sqrt{3}) p_{2}\right)
\end{gathered}
$$




$$
\begin{gathered}
\rho_{32}=\frac{r}{16}\left(-1-i \sqrt{3}+2 \alpha+(1+3 i \sqrt{3}+(-5-i \sqrt{3}) \alpha) p_{1}+(3+i \sqrt{3}-3 \alpha+i \sqrt{3} \alpha) p_{2}+\right. \\
\left.(\sqrt{3}(2-\alpha) i-3 \alpha) p_{4}+(\sqrt{3}(1+\alpha) i+3(1-\alpha)) p_{5}\right) \\
\rho_{33}=-\frac{r}{16}\left(-2+2 p_{1}+(3-i \sqrt{3}) p_{4}+(3+i \sqrt{3}) p_{5}\right)
\end{gathered}
$$

where it is easy to work out the probability of getting a state $|2\rangle$ at the end of the game, and equal with $\frac{1}{8}(-8+(7+\alpha) r)\left(-1+p_{1}\right)$.

\section{Conclusion}

We have introduced the method to discuss the quantum game of roulette with arbitrary $N$ state. In this method, we can get a matrix $D$ which the density matrix of initial state of roulette can be changed to after Bob using a proper unitary transformation $U_{1}$, that is $\rho_{1}=D$. And then, Bob can use another proper unitary transformation $U_{2}$ to control the game because this matrix $D$ is invariant under the classical transposition. This method is general and one can employ to any finite-dimensional quantum game. Finally, we have considered the interaction between the system and its environment and investigate depolarizing channel on this strategy. In this case, we have shown that if Bob cannot control the noise in the system completely, he stands to lose the advantage through the utilization of quantum devices.

\section{References}

[1] D. R. Simon (1994), On the power of quantum computaion, in Proceeding edings of the 35th Symposium on Foundations of Computer Science, Santa Fe, NM, 20-22 November (Los Alamitos, CA: IEE Computer Society Pres) 116-123.

[2] P. W. Shor(1994), Algorithms for quantum computaion: discret logarithms and factoring, in Procedings of the 35th Symposium on Foundations of Computer Science, Santa Fe, S.Goldwaser(ed.), Los Alamitos, CA: IEE Computer Society Pres) 124-134. 
[3] C. H. Benet and G. Brasrd(1984), Quantum cryptography : Public-key distribution and coin tossing,in Procedings of the IEEE International Confernce on Computers, Systems and Signal Proceesing, Bangalore, India, December 1984 (New York: IEEE 1984)175-179.

[4] A. Ekert, Phys. Rev. Lett. 67 (1991) 661-663.

[5] D. A. Meyer, Phys. Rev. Lett. 82, 1052 (1999).

[6] L. Goldenberg, L. Vaidman, and S. Wiesner, Phys. Rev. Lett. 82, 3356 (1999).

[7] Y. S. Zhang, C. F. Li, and et al. quant-ph/0001008 (2000).

[8] J. Eisert, M. Wilkens, and M. Lewenstein, Phys. Rev. Lett. 83, 3077 (1999).

[9] S. C. Benjamin, and P.M. Hayden, Phys. Rev. A 64(3), 030301 (2001).

[10] E. W. Piotrowski, quant-ph/0211191 (2002).

[11] G. Gutoski, arXiv:cs.CC/0511017 (2005).

[12] J. Orlin Grabbe, quant-ph/0506219 (2005).

[13] E. W. Piotrowski, and J. Sladkowski, Int. J. Theor. Phys. 42, 10891099 (2003).

[14] E. Ahmed, M.F. Elettreby, and A.S. Hegaziet, Int. J. Theor. Phys. 45, 880886 (2006).

[15] E. W. Piotrowski, and J. Sladkowski, Int. J. Theor. Phys. 42, 11011106 (2003).

[16] H. F. Ren, and Q. L. Wang, Int. J. Theor. Phys. 47, 18281835 (2008).

[17] R. D. Luce, and H. Raiffa, Games and Decisions. Dover, New York (1989).

[18] X. B. Wang, L.C. Kwek, and C.H. Oh, Phys. Lett. A 278, 4446 (2000).

[19] J. L. Chen, L.C. Kwek, and C.H. Oh, Phys. Rev. A 65, 052320 (2002). 
[20] B. E. Sagan (2001), The symmetric group: representation, combinatorial algorithms, and symmetric function (Springer; 2nd ed. Edition).

[21] F. Zhang(1999), Matrix Theory (Springer-Verlag New York Inc).

[22] B. W. Schumacher, Phys. Rev. 54, 2614 (1996).

[23] K. Kraus (1983), States, Effects and Operations: Fundamental Notions of Quantum Theory (Sqringer-Verlag, Berlin).

[24] A. Barg, IEEE Transaction on Information Theory, Vol. 48, No. 12, 3096-3100 (2002). 